\title{
Label-free in situ imaging of lignification in the cell wall of low lignin transgenic Populus trichocarpa
}

\author{
M. Schmidt • A. M. Schwartzberg • P. N. Perera • A. Weber-Bargioni • A. Carroll • \\ P. Sarkar · E. Bosneaga · J. J. Urban · J. Song $\cdot$ M. Y. Balakshin · E. A. Capanema \\ M. Auer $\cdot$ P. D. Adams $\cdot$ V. L. Chiang $\cdot$ P. James Schuck
}

Received: 1 May 2009 / Accepted: 25 May 2009 / Published online: 13 June 2009

(C) The Author(s) 2009. This article is published with open access at Springerlink.com

\begin{abstract}
Chemical imaging by confocal Raman microscopy has been used for the visualization of the cellulose and lignin distribution in wood cell walls. Lignin reduction in wood can be achieved by, for example, transgenic suppression of a monolignol biosynthesis gene encoding 4-coumarate-CoA ligase (4CL). Here, we use confocal Raman microscopy to compare lignification in wild type and lignin-reduced 4CL transgenic Populus trichocarpa stem wood with spatial resolution that is sub- $\mu \mathrm{m}$. Analyzing the lignin Raman bands in the spectral region between 1,600 and $1,700 \mathrm{~cm}^{-1}$, differences in lignin signal intensity and localization are mapped in situ. Transgenic reduction of lignin is particularly pronounced in the S2 wall layer of fibers, suggesting that such transgenic approach may help overcome cell wall recalcitrance to wood saccharification.
\end{abstract}

Electronic supplementary material The online version of this article (doi:10.1007/s00425-009-0963-x) contains supplementary material, which is available to authorized users.

M. Schmidt · P. N. Perera · A. Carroll · P. Sarkar · E. Bosneaga Energy Biosciences Institute, University of California,

Berkeley, CA 94720, USA

A. M. Schwartzberg - A. Weber-Bargioni - J. J. Urban ·

P. J. Schuck $(\square)$

Molecular Foundry, Lawrence Berkeley National Laboratory,

Berkeley, CA 94720, USA

e-mail: PJSchuck@lbl.gov

\section{A. Carroll}

Department of Biology,

Stanford University, Stanford, CA 94305, USA

J. Song · M. Y. Balakshin · E. A. Capanema · V. L. Chiang Forest Biotechnology Group,

Department of Forestry and Environmental Resources, College of Natural Resources, North Carolina State University,

Raleigh, NC 27695, USA
Spatial heterogeneity in the lignin composition, in particular with regard to ethylenic residues, is observed in both samples.

Keywords Populus trichocarpa (black cottonwood) Cell wall $\cdot$ Lignin $\cdot$ Microspectroscopy $\cdot$ Raman imaging · Transgenic

\section{Introduction}

Plant cell walls are the most abundant renewable resource of photosynthetically fixed carbon. This lignocellulosic biomass is poised to become the primary source for the production of liquid biofuels (Herrera 2006; Himmel et al. 2007; Pauly and Keegstra 2008a, b; Ragauskas et al. 2006; Somerville 2007). The recalcitrance of plant cell wall

M. Auer

Life Sciences Division,

Lawrence Berkeley National Laboratory,

Berkeley, CA 94720, USA

P. D. Adams

Physical Biosciences Division,

Lawrence Berkeley National Laboratory,

Berkeley, CA 94720, USA

Present Address:

J. Song

Institute of Medicinal Plant Development,

Peking Union Medical College,

Chinese Academy of Medical Sciences, Xibeiwang,

Haidian District, 100094 Beijing, People's Republic of China 
materials to cost-effective and efficient degradation, however, poses a major impediment for their use in the production of transportation fuels and chemicals (Pauly and Keegstra 2008b). Plant cell walls have a heterogeneous and complex hierarchical structure, which is primarily made up of cellulose, hemicelluloses, and lignin. The detailed structure of plant cell walls, especially at the ultrastructural level, however, remains to be fully elucidated. Yet, such a detailed knowledge is required to understand the specific chemical and physical obstacles to breakdown.

Lignin is a complex and irregular poly-phenylpropanoid heteropolymer present in the cell walls of vascular plants, and, after cellulose, is the second most abundant natural polymer (Boerjan et al. 2003; Dixon and Reddy 2003; Fukushima 2001; Ralph et al. 2007). Lignin is essential for structural integrity of the cell wall and stiffness and strength of the stem and root; it waterproofs the wall enabling transport of water and solutes through the vascular system, and it is involved in protecting plants against pathogens. As a direct result of the chemical and structural properties tailored for these functional roles, lignin in particular becomes problematic to the postharvest deconstruction of lignocellulosic biomass. For example, in biomass conversion for biofuels, lignin inhibits saccharification in processes aimed at producing simple sugars for fermentation to ethanol (Ralph et al. 2007). The effective use of plants for industrial purposes is in fact largely dependent on the extent to which the plant cell wall is lignified. Importantly, the removal of lignin is a costly and limiting factor (Chiang 2002). Therefore, lignin has become a key plant breeding and genetic engineering target in order to improve cell wall conversion.

Wood is one of the possible feedstocks for the commercial production of cellulosic ethanol (Herrera 2006; Ragauskas et al. 2006). Genetic engineering of trees has generated substantial interests aiming at the development of genotypes with improved wood traits. In wood of transgenic Populus tremuloides, reduction of lignin has been achieved through the suppression of the monolignol pathway gene encoding 4-coumarate-CoA ligase (4CL) by antisense inhibition (Hu et al. 1999). Low lignin wood offers potentials for improved biomass conversion into fermentable sugars.

Analytical tools that permit the accurate rapid characterization of plant cell walls become increasingly important for evaluating specific traits associated with a large number of breeding populations. Accurate investigation of specific chemical characteristics of lignocellulosic materials can be difficult since extractive procedures for the isolation of native components are inevitably destructive, bringing about significant chemical and structural modifications (Atalla and Agarwal 1985, 1986; Fukushima 2001). Analytical chemical in situ methods are, thus, invaluable tools for the compositional and structural characterization of lig- nocellulosic materials. Some of these techniques, e.g., highresolution solution-state nuclear magnetic resonance (Yelle et al. 2008), near-infrared (NIR), mid-infrared, and Raman spectroscopy, can afford non-destructive and comparatively inexpensive measurements with minimal sample preparation. NIR spectroscopy was introduced as a rapid analysis tool for characterizing the bulk chemical properties of transgenic aspen wood (Yamada et al. 2006). Most recently, the rapid analysis of lignin monomer composition in hybrid poplar by a streamlined thioacidolysis procedure and NIR reflectance-based prediction modeling was established enabling medium- to high-throughput screening (Robinson and Mansfield 2009). Raman spectroscopy was shown to be well suited to give insights into both the composition and the structure of lignocellulosic materials (Agarwal 1999, 2008; Agarwal and Ralph 1997; Atalla and Agarwal 1985, 1986); it has multi-phase sensitivity and it is label-free and non-invasive in nature. Raman spectroscopy enabled the study of changes in lignin structure in the xylem of stems of genetically manipulated tobacco plants (Stewart et al. 1997). It also permitted in situ quantification of the aromatic-ring-conjugated ethylenic structures of lignin in spruce wood (Agarwal and Ralph 2008). Raman microscopy was used on wood samples, coupling the spectral analysis with spatial resolution (Atalla and Agarwal 1985, 1986; Roder et al. 2004; Roder and Sixta 2005). Confocal Raman microscopy facilitated the chemical imaging of poplar (Gierlinger and Burgert 2006; Gierlinger and Schwanninger 2006) and spruce wood cell walls (Agarwal 2006). It enabled the in situ study of the spatial distribution and orientation of cell wall polymers, in particular cellulose and lignin. The potential of Raman microscopy and Raman imaging in plant research was discussed in detail in a recent review (Gierlinger and Schwanninger 2007).

Here, we use confocal Raman microscopy to acquire and compare chemical images of wild type and lignin-deficient transgenic Populus trichocarpa (black cottonwood) wood cross-sections. Differences in lignin content, localization, and chemical composition in the cell walls are visualized without staining or labeling of the tissues.

\section{Materials and methods}

Samples and sample preparation

A cDNA (Ptr4CL3, GenBank accession number: EU603298) encoding lignin specific 4-coumarate-CoA ligase (Hu et al. 1999) was cloned from stem differentiating xylem of $P$. trichocarpa (black cottonwood genotype Nisqually-1). The coding sequence of Ptr $4 C L 3$ was cloned into a modified binary vector $\mathrm{pBI} 121$ in an antisense orientation with respect to a CaMV double $35 \mathrm{~S}$ promoter to replace the $G U S$ 
gene. The binary vector was then mobilized into Agrobacterium tumefaciens $\mathrm{C}-58$ for transforming Nisqually-1 following Song et al. (2006). Greenhouse grown transgenic and untransformed P. trichocarpa (Nisqually-1) wild-type plants of 8 months old were quantified for stem wood chemical composition (Hu et al. 1999; Yamada et al. 2006). Stem wood from two transgenic $P$. trichocarpa lines, as4CL-12 and as4CL-4, and a wild-type control, having lignin contents of $12.1,15.0$ and $20.5 \%$, respectively, was used for this study. The lignin content represents the total quantity of the acid-insoluble and acid-soluble lignin determined by the Klason method (Hu et al. 1999; Yamada et al. 2006). For comparison, a fragment of a branch of a 7 -yearold Populus angustifolia tree, grown in the University of California at Berkeley Botanical Garden, was obtained. $100-\mu \mathrm{m}$-thick transverse sections from the native woody tissues were prepared by microtome (Leica, VT1000 S vibrating-blade microtome). The sections were air-dried, placed in $\mathrm{D}_{2} \mathrm{O}$ (Aldrich) on glass microscope slides and covered with glass cover slips $(18 \times 18 \times 0.17 \mathrm{~mm})$. The edges of the cover slips were sealed onto the glass slides to prevent evaporation of $\mathrm{D}_{2} \mathrm{O}$.

Raman analysis

Two-dimensional spectral maps were acquired with a confocal Raman microscope (WITec, alpha300 S, fiber/pinhole diameter $=100 \mu \mathrm{m})$ equipped with a piezoelectric scan stage. In order to gain high spatial resolution, a $100 \times$ oil immersion microscope objective (Nikon, $\mathrm{NA}=1.40$, $\mathrm{WD}=0.13 \mathrm{~mm}$ ) and a laser in the visible wavelength range $(\lambda=532 \mathrm{~nm})$ were used. The linearly polarized laser light was focused with a nearly diffraction-limited spot size onto the samples and the Raman light was detected by a CCD camera (Andor, DV401-BV) behind a grating (600 grooves $\mathrm{mm}^{-1}$ ) spectrometer (WITec, UHTS 300) with a spectral resolution of $3 \mathrm{~cm}^{-1}$. The laser power on the samples was approximately $30 \mathrm{~mW}$. The lateral resolution of our system was determined via a knife-edge measurement within our sample fluid cell to be $\sim 300 \mathrm{~nm}$, which is not significantly lower than the theoretical limit $(0.61 \lambda / \mathrm{NA} \approx 230 \mathrm{~nm})$. Sample areas of $40 \times 40 \mu \mathrm{m}^{2}$ were mapped in 200-nm steps recording 40000 spectra with an integration time of $100 \mathrm{~ms}$ per spectrum. The WITec Project software (version 1.94) and MATLAB (MathWorks, version 7.7) were used for spectral and image processing and analysis. Chemical images were computed from the two-dimensional spectral maps by integrating the intensity over a defined wavenumber range in the baseline-corrected Raman spectra and by evaluating the area under fitted Gaussian peaks of selected bands. Intensity normalization between the different spectral maps was performed using as a reference the peak height of the O-D stretching band around $2,500 \mathrm{~cm}^{-1}$
(Atalla and Agarwal 1985; 1986) in the average lumen spectra (obtained from k-means clustering classification), for which the observed variation was $\leq 6 \%$.

\section{Results}

The average Raman spectra of wild type and as4CL-12 transgenic $P$. trichocarpa, recorded from $40 \times 40 \mu \mathrm{m}^{2}$ sample areas of cross-sections in $\mathrm{D}_{2} \mathrm{O}$ and normalized with respect to the O-D stretching band, are presented in Fig. 1. The Raman bands are attributable primarily to the major wood polymers found in poplar, i.e., cellulose, xylan, glucomannan, and lignin. Band assignments are given in the literature (Agarwal 1999; Agarwal and Ralph 1997; Atalla and Agarwal 1986; Gierlinger and Schwanninger 2006; Himmelsbach et al. 1999). It is noted that in the region between 1,600 and $1,700 \mathrm{~cm}^{-1}$ the vastly dominant spectral contribution stems from lignin. There are three peaks at $1,605,1,628$, and $1,665 \mathrm{~cm}^{-1}$, highlighted in the inset of Fig. 1, which are useful markers of lignin (Agarwal 1999) (Table 1). These lignin bands are significantly reduced in intensity in the as4CL-12 transgenic wood compared to the wild type. It was shown that the relative spectral contributions of different lignin units are not only dependent on concentration but also on pre-resonance Raman and conjugation effects (Agarwal 1999). The magnitude of the intensity decrease in all three bands, however, is clearly indicative of an overall reduction in lignin content in as4CL-12, which is consistent with the chemical analysis. By contrast, the overall carbon content, estimated from the

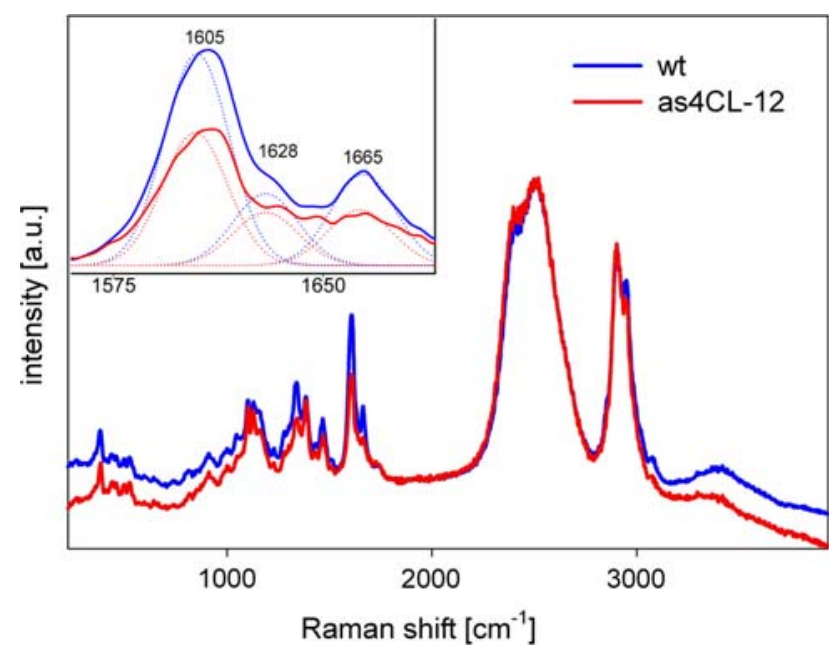

Fig. 1 The average Raman spectra of wild type (wt) and as4CL-12 transgenic Populus trichocarpa, recorded from $40 \times 40 \mu \mathrm{m}^{2}$ sample areas of cross-sections in $\mathrm{D}_{2} \mathrm{O}$ and normalized with respect to the $\mathrm{O}-\mathrm{D}$ stretching band. Inset spectral region having three peaks specifically attributable to lignin. Deconvolved Gaussian fits to the spectra are also shown (dotted lines) 
Table 1 Assignment of selected bands in the Raman spectra of poplar

\begin{tabular}{ll}
\hline Band $\left(\mathrm{cm}^{-1}\right)$ & Assignment $^{\mathrm{a}}$ \\
\hline 1,605 & Symmetric aryl ring stretching \\
1,628 & Ring-conjugated $\mathrm{C}=\mathrm{C}$ stretch of coniferaldehyde \\
1,665 & $\begin{array}{l}\text { Ring-conjugated } \mathrm{C}=\mathrm{C} \text { stretch of coniferyl alcohol; } \\
\mathrm{C}=\mathrm{O} \text { stretch of coniferaldehyde }\end{array}$
\end{tabular}

${ }^{a}$ According to the literature (Agarwal 1999; Agarwal and Ralph 2008)

C-H stretching bands between 2,800 and $3,100 \mathrm{~cm}^{-1}$, is comparable for both samples. These results form the basis for a more in-depth analysis of the spatial distribution of lignin as a result of genetic reduction.

To obtain chemical images, position-resolved microspectroscopic measurements were performed acquiring two-dimensional spectral maps. The basic morphology of the measured cell walls in the wild type (Fig. 2a) and as4CL-12 (Fig. 2d) becomes apparent in the chemical images based on the composite $\mathrm{C}-\mathrm{H}$ stretching bands, i.e., integrating the intensity over the range from 2,775 to $3,125 \mathrm{~cm}^{-1}$. The spatial distribution of lignin is visualized by integrating the intensity from 1,550 to $1,700 \mathrm{~cm}^{-1}$ (Fig. 2b, e). In wild-type wood, there is strong contrast between morphologically distinct cell wall regions due to different lignin signal intensity. High lignin signal intensity is observed in the cell corners (CC) and, somewhat less, in the compound middle lamellae (CML) (Fig. 2b). The results are consistent with earlier studies of lignin distribution in the xylem of gymnosperms and angiosperms by Raman microscopy (Agarwal 2006; Gierlinger and Burgert 2006; Gierlinger and Schwanninger 2006), UV microscopy (Koch and Kleist 2001), energy-dispersive X-ray analysis (Westermark et al. 1988), and interference and fluorescence microscopy (Donaldson et al. 2001). Within the S2 wall layer of the fibers less, yet not insubstantial, amounts of lignin are observed. The S2 layer of vessels shows stronger lignin signal than the $\mathrm{S} 2$ layer of fibers, as noted previously (Gierlinger and Burgert 2006). Lignin intensity and distribution measured in wood of $P$. angustifolia gave very similar results (data not shown).

By contrast, there are marked differences for the as $4 \mathrm{CL}$ 12 wood (Fig. 2e). While the relative lignin signal intensity is still higher in the CC and CML than in the S2 layer, there is a clear overall reduction of lignin compared to the wild type. In the S2 wall layer of the fibers, lignin is significantly depleted. This is further illustrated by the comparison of the $\mathrm{C}-\mathrm{H}$ and lignin band intensity profiles of line cuts across cell walls in the wild type and as4CL-12 (Fig. 2c, f). Measurements on a second transgenic line, as4CL-4 (15.0\% lignin content), gave comparable results to those of as $4 \mathrm{CL}-12$ (Supplementary Figure).
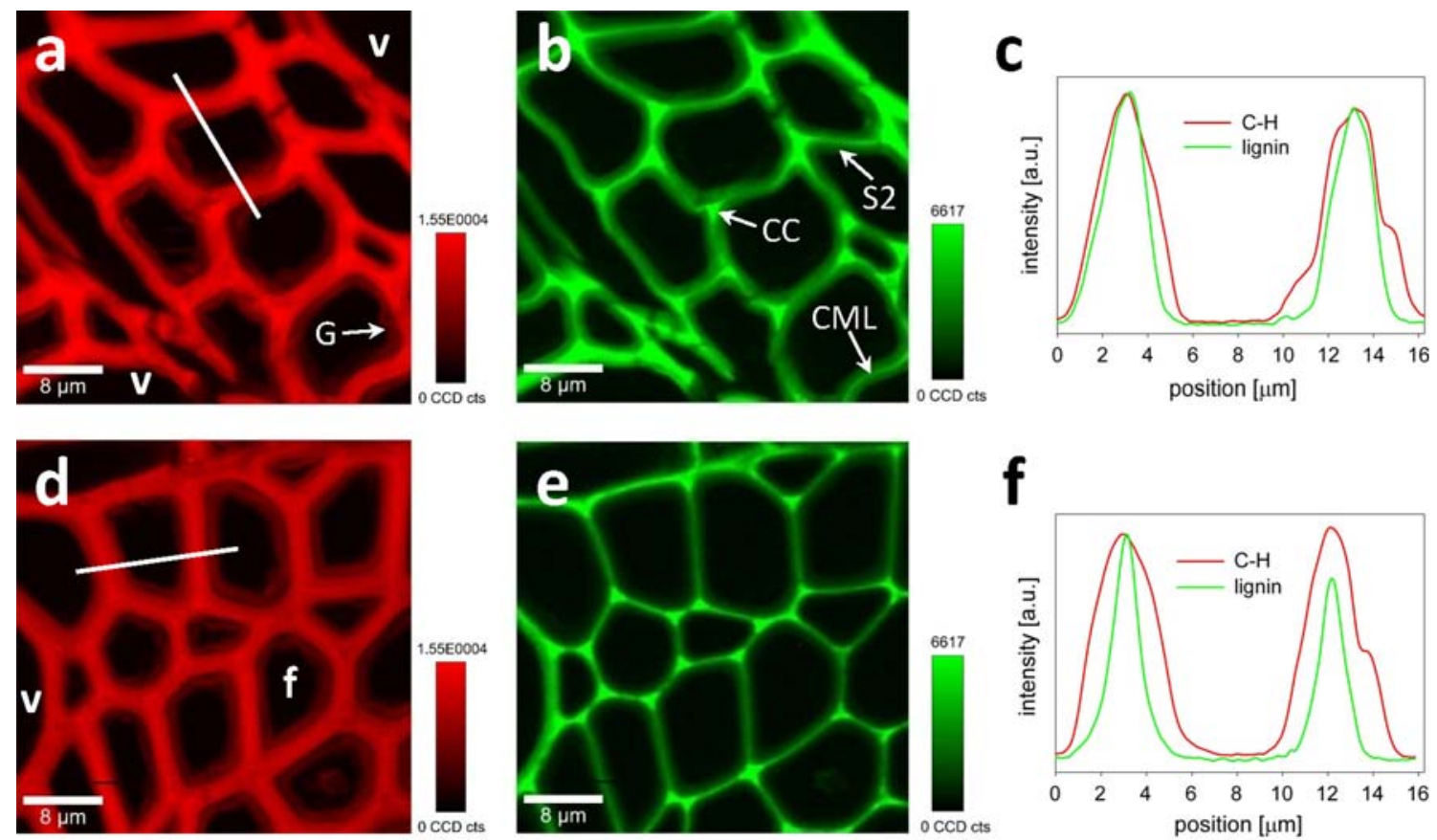

Fig. 2 Chemical images based on the composite $\mathrm{C}-\mathrm{H}$ stretching bands (integrating the intensity from 2,775 to $3,125 \mathrm{~cm}^{-1}$ ) for wild type (a) and as4CL-12 (d). $v$ vessel, $G$ gelatinous layer (G-layer), $f$ fiber. b Lignin distribution (integrating from 1,550 to $1,700 \mathrm{~cm}^{-1}$ ) in wild type. $C C$ cell corner, $C M L$ compound middle lamella, $S 2 \mathrm{~S} 2$ sublayer of the secondary cell wall. e For comparison, lignin distribution in as4CL-12. Normalized $\mathrm{C}-\mathrm{H}$ and lignin band intensity profiles of line cuts across cell walls in wild type (c) and as4CL-12 (f), corresponding to the thin white lines in (a) and (d), respectively 
Owing to the in situ chemical sensitivity and the specific chemical attributions of the lignin bands (Table 1) (Agarwal and Ralph 2008), it is possible to further dissect the spatial lignin distribution both within and between samples. While the $1,605 \mathrm{~cm}^{-1}$ band is due to aryl ring stretching and thus is a more general lignin marker, the $1,628 \mathrm{~cm}^{-1}$ band is indicative of coniferaldehyde and the $1,665 \mathrm{~cm}^{-1}$ band of coniferyl alcohol and coniferaldehyde (Agarwal 1999; Agarwal and Ralph 2008). For the wild-type sample, the chemical image based on the $1,605 \mathrm{~cm}^{-1}$ band is shown in Fig. 3a.
The highest lignin signal intensity is observed in $\mathrm{CC}$, followed by CML and the cell walls of vessels. Also, variability of lignin signal intensity is found to some extent within CC, CML and S2, especially from fiber to fiber. There is substantial similarity between the spatial distributions of the 1,628 and $1,665 \mathrm{~cm}^{-1}$ bands (data not shown) and that of the $1,605 \mathrm{~cm}^{-1}$ band, especially for the $1,665 \mathrm{~cm}^{-1}$ band and somewhat less for the $1,628 \mathrm{~cm}^{-1}$ band. In order to glean more subtle differences, images for band ratios were obtained. In the image showing the ratio of the $1,628 \mathrm{~cm}^{-1}$
Fig. 3 Chemical images based on the deconvolved lignin band at $1,605 \mathrm{~cm}^{-1}$ for wild type (a) and as4CL-12 (b). Images for the ratio of the $1,628 \mathrm{~cm}^{-1}$ to the $1,605 \mathrm{~cm}^{-1}$ band, showing spatially distinct chemical composition of lignin in wild type $(\mathbf{c})$ and as4CL-12 (d). For comparison, images for the ratio of the $1,665 \mathrm{~cm}^{-1}$ to the $1,605 \mathrm{~cm}^{-1}$ band of lignin in wild type (e) and as4CL-12 (f)
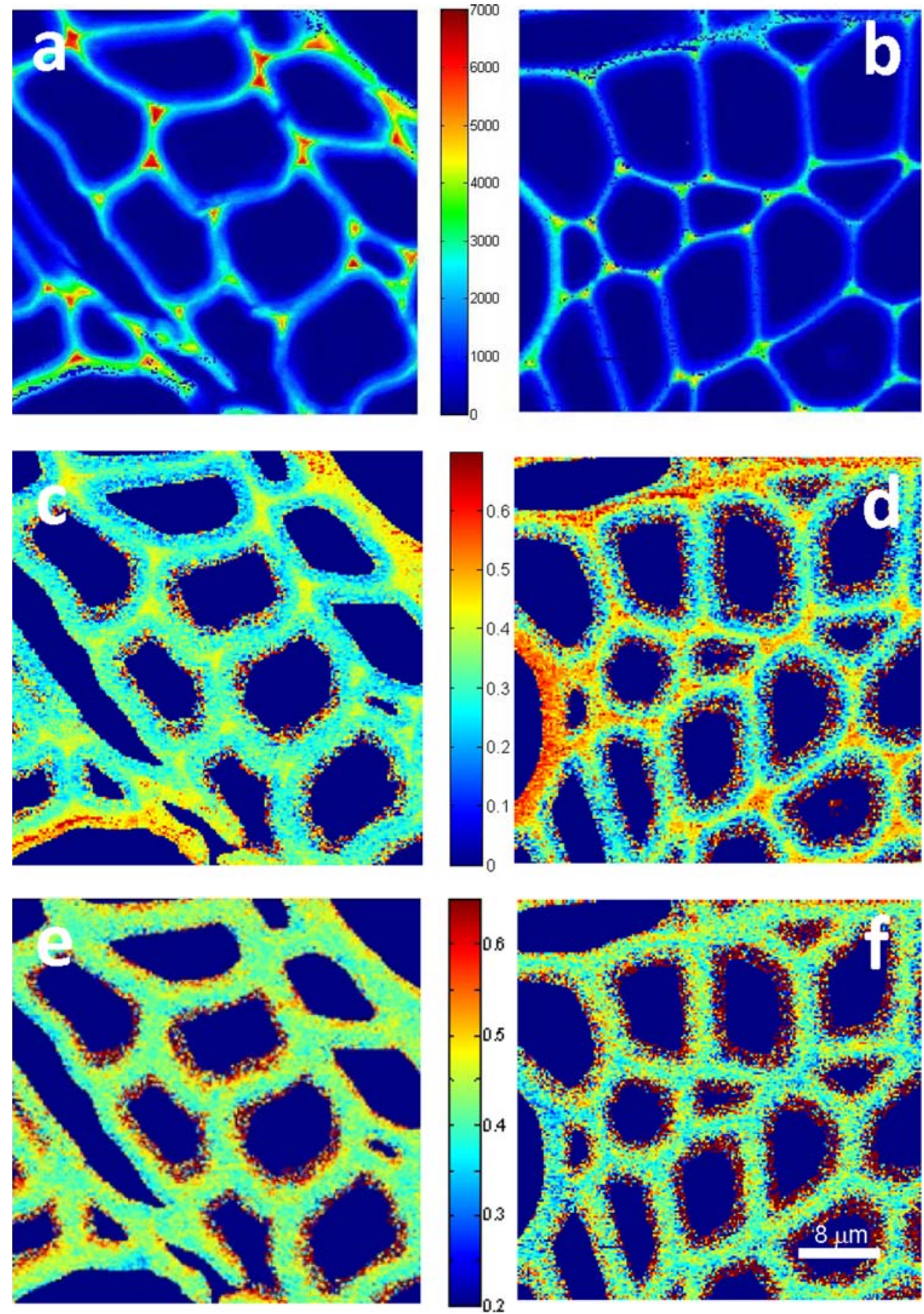
Fig. 4 Chemical images based on the O-D stretching band (a) (integrating the intensity from 2,200 to $2,775 \mathrm{~cm}^{-1}$ ) and the $\mathrm{O}$ $\mathrm{H}$ stretching band (b) (integrating from 3,200 to $3,800 \mathrm{~cm}^{-1}$ ) for as 4 CL-12. $\mathbf{c}$ Image for the cellulose-orientation-sensitive band at $1,096 \mathrm{~cm}^{-1}$ (integrating from 1,080 to $1,115 \mathrm{~cm}^{-1}$ ) visualizing the $\mathrm{S} 1$ sublayer of the secondary cell wall parallel to the electric field vector of the laser beam. $\mathbf{d}$ The average G-layer spectra of wild type (wt) and as4CL-12. Inset spectral region with three lignin-specific peaks
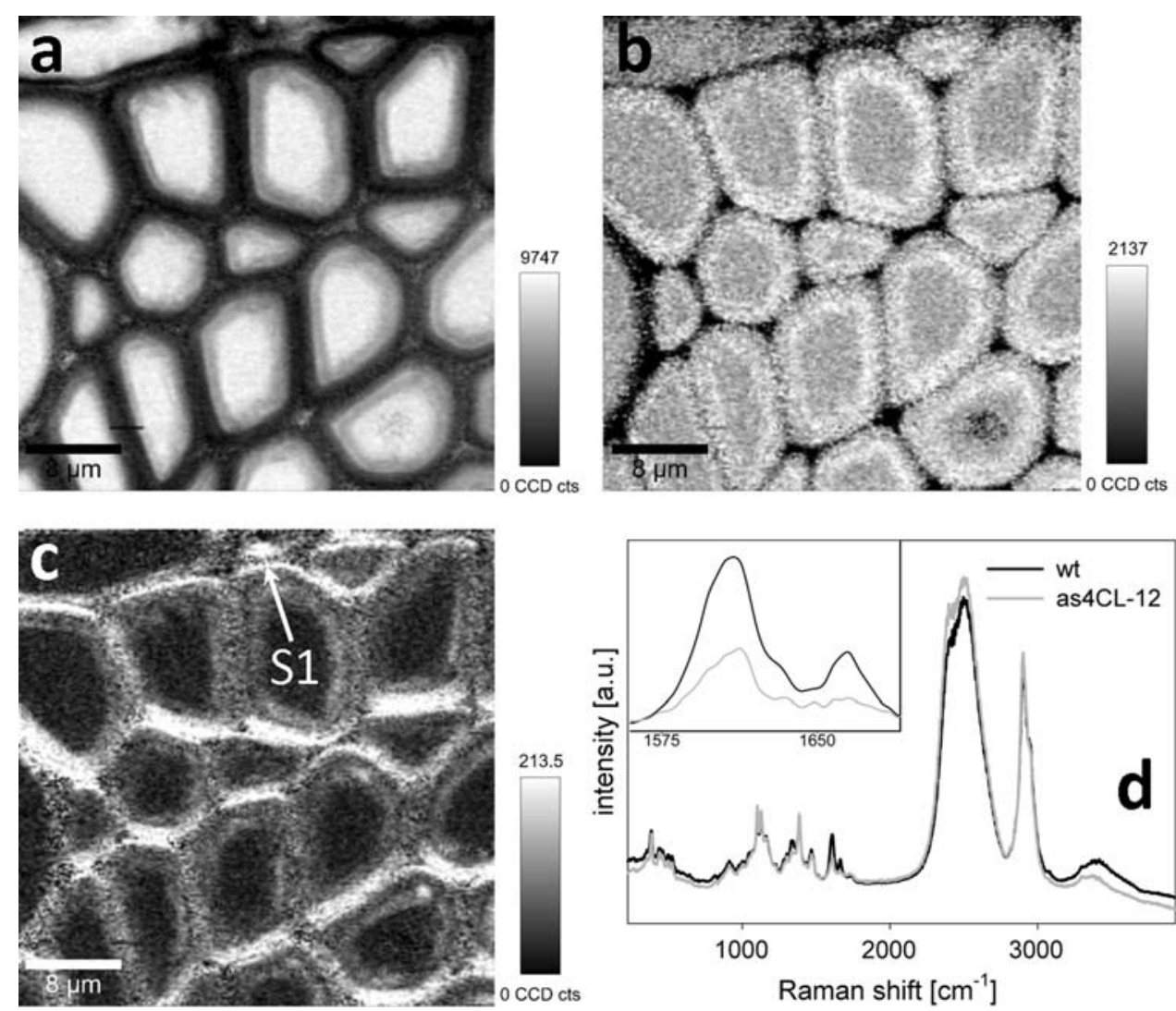

to the $1,605 \mathrm{~cm}^{-1}$ band (Fig. 3c), a relative increase of coniferaldehyde is observed, especially in the cell walls of vessels and, to a lesser degree, in $\mathrm{CC}$. The heterogeneity in the band ratio intensity is a direct evidence of the spatially distinct chemical composition of lignin. In the image for the ratio of the $1,665 \mathrm{~cm}^{-1}$ to the $1,605 \mathrm{~cm}^{-1}$ band (Fig. 3e), however, the intensity distribution is much more homogeneous, suggesting a compensatory effect of coniferyl alcohol and coniferaldehyde contents or less spectral sensitivity. The chemical image for as4CL-12 based on the $1,605 \mathrm{~cm}^{-1}$ band is shown in Fig. 3b. There is a marked reduction in intensity for this band, and also for the 1,628 and $1,665 \mathrm{~cm}^{-1}$ bands (data not shown), compared to the wild type. This is indicative of overall less lignin as well as less coniferyl alcohol and coniferaldehyde content. The spatial heterogeneity seen in the image of the ratio of the $1,628 \mathrm{~cm}^{-1}$ to the $1,605 \mathrm{~cm}^{-1}$ band (Fig. 3d) is much more pronounced than for the wild type, with high relative coniferaldehyde signal in the $\mathrm{S} 2$ layer of the vessel wall and also in CC and CML. The intensity distribution of the $1,665 \mathrm{~cm}^{-1}$ to the $1,605 \mathrm{~cm}^{-1}$ band ratio (Fig. 3f) is relatively homogeneous.

Measuring woody samples in $\mathrm{D}_{2} \mathrm{O}$ has been previously shown to be advantageous because it limits laser-induced fluorescence, provides a convenient internal reference for intensity normalization, and minimizes overlap between the O-H and C-H stretching bands (Atalla and Agarwal 1985, 1986). As an extrinsic marker, $\mathrm{D}_{2} \mathrm{O}$ can give additional chemical contrast and insights into accessibility and reactivity (H-D exchange) of different structural parts (Schmidt et al. 2006). In Fig. 4a the chemical image based on the O-D stretching band (integrating the intensity from 2,200 to $2,775 \mathrm{~cm}^{-1}$ ) is shown for as4CL-12. As expected, high intensity is observed in the lumen. The O-D signal, albeit much weaker, is also observed in the S2 wall layer, the CC and in some parts of the CML. This may be explained in terms of the hydrophilicity or porosity of the various structures. For comparison, the chemical image based on the $\mathrm{O}-\mathrm{H}$ stretching band (integrating the intensity from 3,200 to $3,800 \mathrm{~cm}^{-1}$ ), attributable to hydroxyl groups in carbohydrates inaccessible to $\mathrm{D}_{2} \mathrm{O}$, as well as residual and generated $\mathrm{H}_{2} \mathrm{O}$ (and $\mathrm{HDO}$ ), is shown in Fig. 4b. Little or no signal could be detected in the $\mathrm{CC}$ and CML. As was reported earlier (Gierlinger and Burgert 2006; Gierlinger and Schwanninger 2006), it is possible to visualize the S1 layer in the walls parallel to the electric field vector of the laser beam, being in the horizontal direction in the given case, by examining the cellulose-orientation-sensitive band at $1,096 \mathrm{~cm}^{-1}$ (integrating the intensity from 1,080 to $1,115 \mathrm{~cm}^{-1}$ ) (Fig. 4c).

A minor G-layer is discernible towards the lumen in a number of fibers (Fig. $2 \mathrm{a}, \mathrm{d}$ ). The gelatinous layer or G-layer, which is mainly constituted of cellulose microfibrils oriented nearly parallel to the fiber axis, is an extra secondary wall layer in tension wood in a number of hardwood 
species. G-layers can always be observed in wood of trees under normal growth conditions; however, the extent of G-layer formation is insignificant compared to true tension wood fibers [cf. (Gierlinger and Schwanninger 2006)] and unlikely to result in noticeable changes in secondary wall biosynthesis in the fiber walls (Pilate et al. 2004). The average G-layer spectra of wild type and as4CL-12 are shown in Fig. 4d. As expected, only very weak lignin signals are detected (cf. Fig. 1), with the spectral patterns of the lignin bands (inset in Fig. 4d) resembling those observed in the sample average spectra (inset of Fig. 1). However, secondary wall biosynthesis and lignification in true tension wood are known to be different from normal wood (Pilate et al. 2004). Previously, differences in cellulose and lignin distributions in normal and tension poplar wood samples were visualized by confocal Raman microscopy (Gierlinger and Schwanninger 2006). It seems, therefore, that comparing in situ the chemical composition and lignification of normal and tension (G-layer as well as opposite) wood fibers in both wild type and transgenic samples would be an interesting avenue of future investigation.

\section{Discussion}

Confocal Raman microscopy afforded the label-free in situ visualization of the lignification in wild type and transgenic $P$. trichocarpa wood. The combination of chemical sensitivity with spatial resolution made it possible to compare the lignin signal intensity as well as localization. The large intensity differences between the samples, i.e., the reduction of lignin in transgenics, and also between morphologically distinct cell wall regions within the samples do not suggest an effect of lignin orientation, if present (Agarwal 2006). Further analysis also revealed spatial heterogeneity in the lignin composition of both samples. Raman spectroscopy has a high sensitivity for detecting ring-conjugated ethylenic units (Agarwal 1999; Agarwal and Ralph 2008), which are present at low concentrations mainly as coniferaldehyde and coniferyl alcohol residues. In an earlier study on spruce wood, it was found that the coniferaldehyde and coniferyl alcohol distribution followed that of lignin and that no particular cell wall layer or region was enriched in these ethylenic residues (Agarwal 2006; 2008). In the present case, however, where by contrast vessels were also measured, relative coniferaldehyde enrichment in these vessel walls is observed (Fig. 3c, d). The variability in the relative coniferaldehyde distribution, based on the $1,628-1,605 \mathrm{~cm}^{-1}$ band ratio, is more pronounced in the low-lignin transgenic wood, where relative enrichment is also found in CC and CML of fibers. It is noted, however, that the absolute coniferaldehyde content in as4CL-12 is less than in wild type. Analyzing the relative intensity of the $1,665 \mathrm{~cm}^{-1}$ band (Fig. 3e, f), which is attributed to both, coniferyl alcohol and coniferaldehyde, the respective intensity distributions are seen to be much more homogeneous. This may be due to a compensatory effect of coniferyl alcohol and coniferaldehyde contents, or the spectral sensitivity to detect small differences may not be adequate for this band. It was shown that secondary walls of vessels generally have higher lignin content than those of fibers, and that lignin in vessels is generally enriched in guaiacyl units, whereas lignin in fibers is typically enriched in syringyl units (Boerjan et al. 2003; Fukushima 2001; Ralph et al. 2007). Vessels, especially, are prone to buckle under mechanical load and to collapse. The relative coniferaldehyde enrichment observed in the vessel walls may contribute to the mechanical strengthening of these composite walls, conceivably via hydrogen bonding to cellulose and hemicelluloses (Agarwal 1999).

The determination of lignin content and composition and other physico-chemical attributes of plant cell walls are of great importance for screening and evaluating the results of plant breeding and genetic engineering. Chemical imaging by confocal Raman microscopy enables the in situ analysis of lignin and the direct visualization of its heterogeneous distribution. Most wood biomass is in the S2 wall layer of fibers, which, therefore, deserves the main focus of attempts to modify wood properties for possible fuel production (Mellerowicz and Sundberg 2008). An overall reduction of lignin was observed, including a significant depletion in the S2 layer of fibers in the as4CL-12 sample compared to the wild type. This reduction may alleviate the recalcitrance towards saccharification of cellulose and hemicelluloses. The Raman spectra contain information not only on lignin but also cellulose and the hemicelluloses in their native environment. A detailed analysis of these components is more complicated due to their chemical and spectroscopic similarities (Agarwal 1999; Agarwal and Ralph 1997; Gierlinger and Schwanninger 2006). A chemometric approach, including spectra from pure reference compounds and partially extracted wood samples, to dissect the spatial distribution of the carbohydrates is currently underway and will be the subject of a future paper.

Measurements in $\mathrm{D}_{2} \mathrm{O}$ are useful for exploring the porosity and structural accessibilities, important traits that may also affect lignocellulosic conversion efficiency. The technique is amenable to measurements in aqueous environments and conceivably other liquids, such as ionic liquids, which can be suitable solvents of lignocellulosic biomass. Recently, the capabilities of Fourier-transform infrared microscopy for the in situ study on enzymatic treatment of poplar wood cross-sections were demonstrated (Gierlinger et al. 2008). Given the complementary nature and much higher spatial resolution, confocal Raman microscopy holds great potential for dynamic chemical in situ imaging during lignocellulosic conversion. Evaluating the effect of different degrees of lignification in poplar wood on pretreat- 
ment and enzymatic hydrolysis efficiency is a future course of study in our lab. Our current measurement time can be improved by using a spectroscopic electron multiplying CCD camera as a detector (Dieing and Hollricher 2008) or by implementing coherent anti-Stokes Raman scattering (Evans and Xie 2008) and stimulated Raman scattering microscopy (Freudiger et al. 2008) for more sensitive and rapid chemical data collection.

\section{Conclusions}

Based on earlier results showing the spatial distribution of cellulose and lignin in wood cell walls, we used confocal Raman microscopy to compare lignification in wild type and transgenic $P$. trichocarpa wood cross-sections. Examining the lignin Raman bands in the spectral region between 1,600 and $1,700 \mathrm{~cm}^{-1}$, an overall reduction in lignin signal intensity in the as4CL-12 transgenic poplar sample relative to the wild type was detected, which is consistent with the results from a wet chemical analysis. Importantly, differences in lignin localization were visualized in situ in a label-free manner. Lignin was found to be significantly reduced in the S2 wall layers of the transgenic poplar fibers. Given that most wood biomass is in the S2 layer of fibers, this finding may have important implications toward addressing biomass recalcitrance to degradation. By analyzing the three lignin bands at $1,605,1,628$, and $1,665 \mathrm{~cm}^{-1}$ in more detail, it was possible to further dissect the spatial lignin distribution both within and between samples. Spatial heterogeneities in the lignin composition, in particular with regard to coniferaldehyde moieties, were mapped. In both the wild type and transgenic samples, relative coniferaldehyde enrichment was detected, especially in the secondary walls of vessels. The variability in the relative coniferaldehyde distribution was more pronounced in the transgenic sample.

\begin{abstract}
Acknowledgments We thank Shanfa Lu for preparing the transgene expression construct. This work was supported in part by the Energy Biosciences Institute, and the National Research Initiative of the USDA Cooperative State Research, Education and Extension Service (CSREES) \#2004-35504-14625 and \# 2006-35504-17233 to VLC. Work at the Molecular Foundry was supported by the Office of Science, Office of Basic Energy Sciences, of the US Department of Energy under Contract No. DE-AC02-05CH1123.
\end{abstract}

Open Access This article is distributed under the terms of the Creative Commons Attribution Noncommercial License which permits any noncommercial use, distribution, and reproduction in any medium, provided the original author(s) and source are credited.

\section{References}

Agarwal UP (1999) An overview of raman spectroscopy as applied to lignocellulosic materials. In: Argyropoulos DS (ed) Advances in lignocellulosics characterization. TAPPI Press, Atlanta, pp 201225

Agarwal UP (2006) Raman imaging to investigate ultrastructure and composition of plant cell walls: distribution of lignin and cellulose in black spruce wood (Picea mariana). Planta 224:11411153

Agarwal UP (2008) Raman spectroscopic characterization of wood and pulp fibers. In: Hu TQ (ed) Characterization of lignocellulosic materials. Blackwell, Oxford, pp 17-35

Agarwal UP, Ralph SA (1997) FT-Raman spectroscopy of wood: identifying contributions of lignin and carbohydrate polymers in the spectrum of black spruce (Picea mariana). Appl Spectrosc 51:1648-1655

Agarwal UP, Ralph SA (2008) Determination of ethylenic residues in wood and TMP of spruce by FT-Raman spectroscopy. Holzforschung 62:667-675

Atalla RH, Agarwal UP (1985) Raman microprobe evidence for lignin orientation in the cell walls of native woody tissue. Science 227:636-638

Atalla RH, Agarwal UP (1986) Recording Raman spectra from plant cell walls. J Raman Spectrosc 17:229-231

Boerjan W, Ralph J, Baucher M (2003) Lignin biosynthesis. Annu Rev Plant Biol 54:519-546

Chiang VL (2002) From rags to riches. Nat Biotechnol 20:557-558

Dieing T, Hollricher O (2008) High-resolution, high-speed confocal Raman imaging. Vib Spectrosc 48:22-27

Dixon RA, Reddy MSS (2003) Biosynthesis of monolignols. Genomic and reverse genetic approaches. Phytochem Rev 2:289-306

Donaldson L, Hague J, Snell R (2001) Lignin distribution in coppice poplar, linseed and wheat straw. Holzforschung 55:379-385

Evans CL, Xie XS (2008) Coherent anti-stokes Raman scattering microscopy: chemical imaging for biology and medicine. Annu Rev Analyt Chem 1:883-909

Freudiger CW, Min W, Saar BG, Lu S, Holtom GR, He CW, Tsai JC, Kang JX, Xie XS (2008) Label-free biomedical imaging with high sensitivity by stimulated Raman scattering microscopy. Science 322:1857-1861

Fukushima K (2001) Regulation of syringyl to guaiacyl ratio in lignin biosynthesis. J Plant Res 114:499-508

Gierlinger N, Burgert I (2006) Secondary cell wall polymers studied by confocal Raman microscopy: spatial distribution, orientation, and molecular deformation. N Z J For Sci 36:60-71

Gierlinger N, Schwanninger M (2006) Chemical imaging of poplar wood cell walls by confocal Raman microscopy. Plant Physiol 140:1246-1254

Gierlinger N, Schwanninger M (2007) The potential of Raman microscopy and Raman imaging in plant research. Spectrosc Int J 21:6989

Gierlinger N, Goswami L, Schmidt M, Burgert I, Coutand C, Rogge T, Schwanninger M (2008) In situ FT-IR microscopic study on enzymatic treatment of poplar wood cross-sections. Biomacromolecules 9:2194-2201

Herrera S (2006) Bonkers about biofuels. Nat Biotechnol 24:755-760

Himmel ME, Ding SY, Johnson DK, Adney WS, Nimlos MR, Brady JW, Foust TD (2007) Biomass recalcitrance: engineering plants and enzymes for biofuels production. Science 315:804-807

Himmelsbach DS, Khahili S, Akin DE (1999) Near-infrared-Fouriertransform-Raman microspectroscopic imaging of flax stems. Vib Spectrosc 19:361-367

Hu WJ, Harding SA, Lung J, Popko JL, Ralph J, Stokke DD, Tsai CJ, Chiang VL (1999) Repression of lignin biosynthesis promotes cellulose accumulation and growth in transgenic trees. Nat Biotechnol 17:808-812

Koch G, Kleist G (2001) Application of scanning UV microspectrophotometry to localise lignins and phenolic extractives in plant cell walls. Holzforschung 55:563-567 
Mellerowicz EJ, Sundberg B (2008) Wood cell walls: biosynthesis, developmental dynamics and their implications for wood properties. Curr Opin Plant Biol 11:293-300

Pauly M, Keegstra K (2008a) Cell-wall carbohydrates and their modification as a resource for biofuels. Plant J 54:559-568

Pauly M, Keegstra K (2008b) Physiology and metabolism 'tear down this wall'. Curr Opin Plant Biol 11:233-235

Pilate G, Chabbert B, Cathala B, Yoshinaga A, Leple JC, Laurans F, Lapierre C, Ruel K (2004) Lignification and tension wood. C R Biol 327:889-901

Ragauskas AJ, Williams CK, Davison BH, Britovsek G, Cairney J, Eckert CA, Frederick WJ, Hallett JP, Leak DJ, Liotta CL, Mielenz JR, Murphy R, Templer R, Tschaplinski T (2006) The path forward for biofuels and biomaterials. Science 311:484-489

Ralph J, Brunow G, Boerjan W (2007) Lignins. Encyclopedia of life sciences. Wiley, Chichester

Robinson AR, Mansfield SD (2009) Rapid analysis of poplar lignin monomer composition by a streamlined thioacidolysis procedure and near-infrared reflectance-based prediction modeling. Plant $\mathbf{J}$ 58:706-714

Roder T, Sixta H (2005) Confocal Raman spectroscopy-applications on wood, pulp, and cellulose fibres. Macromol Symp 223:57-66

Roder T, Koch G, Sixta H (2004) Application of confocal Raman spectroscopy for the topochemical distribution of lignin and cellulose in plant cell walls of beech wood (Fagus sylvatica L.) compared to UV microspectrophotometry. Holzforschung 58:480-482

Schmidt M, Gierlinger N, Schade U, Rogge T, Grunze M (2006) Polarized infrared microspectroscopy of single spruce fibers: hydrogen bonding in wood polymers. Biopolymers 83:546-555

Somerville C (2007) Biofuels. Curr Biol 17:R115-R119

Song J, Lu S, Chen ZZ, Lourenco R, Chiang VL (2006) Genetic transformation of Populus trichocarpa genotype Nisqually-1: a functional genomic tool for woody plants. Plant Cell Physiol 47:1582-1589

Stewart D, Yahiaoui N, McDougall GJ, Myton K, Marque C, Boudet AM, Haigh J (1997) Fourier-transform infrared and Raman spectroscopic evidence for the incorporation of cinnamaldehydes into the lignin of transgenic tobacco (Nicotiana tabacum L) plants with reduced expression of cinnamyl alcohol dehydrogenase. Planta 201:311-318

Westermark U, Lidbrandt O, Eriksson I (1988) Lignin distribution in spruce (Picea abies) determined by mercerization with SEMEDXA technique. Wood Sci Technol 22:243-250

Yamada T, Yeh TF, Chang HM, Li LG, Kadla JF, Chiang VL (2006) Rapid analysis of transgenic trees using transmittance near-infrared spectroscopy (NIR). Holzforschung 60:24-28

Yelle DJ, Ralph J, Frihart CR (2008) Characterization of non-derivatized plant cell walls using high-resolution solution-state NMR spectroscopy. Magn Reson Chem 46:508-517 